\title{
Why does the Quark-Gluon Plasma at RHIC behave as a nearly ideal fluid?
}

\author{
Edward Shuryak \\ Department of Physics and Astronomy, \\ University at Stony Brook, NY 11794, USA
}

February 1, 2008

\begin{abstract}
The lecture is a brief review of the following topics: (i) collective flow phenomena in heavy ion collisions. The data from RHIC indicate robust collective flows, well described by hydrodynamics with expected Equation of State. The transport properties turned out to be unexpected, with very small viscosity; (ii) physics of highly excited matter produced in heavy ion collisions at $T_{c}<T<4 T_{c}$ is different from weakly coupled quark-gluon plasma because of relatively strong coupling generating bound states of quasiparticles; (iii) wider discussion of other "strongly coupled systems" including strongly coupled supersymmetric theories studied via Maldacena duality, as well as recent progress in trapped atoms with very large scattering length.
\end{abstract}

\section{Introduction}

When high temperature QCD was first addressed, in 1970's (see e.g. [1]), the Quark Gluon Plasma (QGP) was assumed to be a gas of quasiparticles (=dressed quarks and gluons) which interact relatively weakly with each other. All non-perturbative results (such as lattice predictions for Equation of State (EoS)) were not in disagreement with this view. The theoretical work continued on refining the perturbative calculations during 1980's and 1990's, and although it resulted in poorly converging series for realistic $T$, the hopes remained to do some clever resummation and get all the physics right perturbatively.

If that attitude turned to be true, one should also be able to model heavy ion collisions following pQCD, with "parton cascades" limited by some reasonable cutoff around a scale $\sim 1 \mathrm{GeV}$. Many advocates of this approach did the calculations and found very little parton rescattering and essentially no collective effects.

However when the Relativistic Heavy Ion Collider (RHIC) project in Brookhaven National Laboratory was completed and started producing data, from summer of 2000, it became apparent that a number of robust collective effects are observed. This implies that the effective interaction in QGP is in fact much stronger than expected on the basis of the perturbation theory. The data are in fact in much better agreement with the predictions based on ideal hydrodynamics, an effective theory based on the opposite picture of very strong interaction and very small mean free paths ${ }^{1}$. We will discuss below current estimates of the viscosity of QGP, and will see that its ratio to entropy density $\eta / s \sim 1 / 10$ is

\footnotetext{
${ }^{1}$ We will discuss also why the Equation of State calculated perturbatively is still approximately valid.
} 
surprisingly small. In fact, QGP is the most perfect fluid known, since the same ratio is greater than 1 for most liquids ${ }^{2}$.

Let me reformulate the question I put into the title, Why does the Quark-Gluon Plasma at RHIC behave as a nearly ideal fluid? in a more constructive way, by asking instead: What are the QGP properties in the strong coupling regime, when the gauge field coupling $\alpha_{\mathrm{s}}=g^{2} / 4 \pi$ is no longer small but $\sim 1$ or even larger than that?

Our work in this direction has started only recently, and in this lecture I will mostly discuss new ideas pursued in our group. I.Zahed and myself [2] suggested an explanation to hydro regime of QGP, related it with presence of loosely bound pairs of quasiparticles which generate large scattering lengths. These ideas are further developed by Brown et al 3]. However this is just a part of developments in other fields of physics, which happen to be quite recent as well.

One revolutionary advance took place on the theoretical front. String theorists (Maldacena) pointed out that one can treat $\mathcal{N}=4$ supersymmetric gauge field theory in strong coupling regime by considering a weakly coupled supergravity problem in a particular background field. It can be done both at zero and finite $T$, and resulted in a number of fascinating (although rather incomprehensible) results. I will briefly describe those, as well as recent explanations of those, based on deeply bound states recently suggested by I.Zahed and myself [4].

If this development sounds too theoretical for some readers, here is another purely experimental one. Exciting recent development took place at the frontier of low temperature physics, with trapped $\mathrm{Li}^{6}$ (fermionic) atoms. Using magnetic field one can use the so called Feshbach resonances and make a pair of atoms nearly degenerate with their bound state (usually called a molecule but actually a Cooper pair). This results in so large scattering length $a$, than a qualitatively new type of matter - strongly coupled fermi and bose gases - is observed. In particular, this very dilute systems start to behave hydrodynamically, displaying elliptic flow very similar to that in non-central heavy ion collisions.

Following nice traditions of the Erice schools, let me in these lectures go outside nuclear physics and spend some time, providing some introductory discussion into these fascinating fields.

\section{Collective flows and the EoS of QGP}

\subsection{Is hadronic matter really produced in heavy ion collisions?}

Let me start with this central question, asked so often. Is there something qualitatively new in AA collisions, never seen in "elementary" 3 pp or $e+e-$ collisions?

Indeed, the original motivation for heavy ion program is not just increase the number of secondary particles produced per event (up to several thousands at RHIC), but to reach a qualitatively different dynamical regime. characterized by a small microscopic scale $l$ (e.g. mean free path) as compared to the macro scale $L$ (the system's size)

$$
l \ll L
$$

If this is achieved, the fireball produced in heavy ion collisions can be treated as a macroscopic body, and collective "bangs" should be observed, rather than a collision-less fireworks of debris created in a collision seen in $p p$ or $e^{+} e^{-}$. More precisely, if the system is macroscopically large then its description via thermodynamics of its bulk properties (like matter composition) and hydrodynamics for space-time evolution should work.

\footnotetext{
${ }^{2}$ E.g. water, which gave its name to hydrodynamics, does not flow that well if one considers a drop of only $\sim 1000$ molecules.

${ }^{3}$ Apart of the large- $p_{t}$ tail, described by the parton model plus pQCD corrections, it is very far from being elementary and is very poorly understood. One may argue that heavy ion collisions, described well by hydro/thermodynamics, are in fact even much simpler.
} 
Statistical models do indeed work remarkably well for heavy ion collisions, as you will see from other lectures in this school. But it also works for $p p$ or $e^{+} e^{-}$(and we still do not know why). Many years ago, in a prehistoric era before QCD, I have tried to apply hydro to $e^{+} e^{-}$: discovery of jets several years later put the end to it. Also in 1970's, a search for signs of transverse flow in the early hadronic spectra at CERN ISR from pp collisions was performed by (my former student) Zhirov and myself [5] have found little or no signs of the collective transverse flow. Apparent lack of collectivity in $p p$ or $e^{+} e^{-}$ shows that although they also produce a multi-body excited systems, those are not macroscopically large. These collisions do not produce "matter", just a bunch of outgoing particles.

The heavy ion collisions, on the other hand, show collective new phenomena, a variety of "flows" to be discussed. These various forms of a collective expansion confirm that we do see a macroscopic behavior, which can indeed be described using bulk properties of "matter". Although similar "flows" are observed at all energies, at AGS and below they have somewhat different origin, and I would not discuss those for lack of time. I will discuss mostly recent data from RHIC, which prove the point in a spectacular way.

\subsection{The transverse flow}

Let me start with historic comments. The first application of hydrodynamics for description of hadronic fireballs was suggested in the classical work by L.D.Landau 50 years ago [6], two years after the pioneer work on statistical model by Fermi. Landau has aimed his hydrodynamical theory mainly at description of the longitudinal momenta. However with the discovery of asymptotic freedom, Fermi's idea of complete stopping - the initial condition in form of Lorentz contracted disk of equilibrated matter at rest - became problematic ${ }^{4}$. Actually we do not yet know the pre-hydro initial stage well even now, so longitudinal hydro has little predictive power.

First attempts to connect the experimental information with the collective transverse flow were made independently by Siemens and Rasmussen [8] for low energy (BEVALAC) and by Zhirov and myself [5] for high energy pp collisions at CERN ISR. The idea was exactly the same: the collective velocity of explosion boosts spectra of light and heavy particles differently. Pions are ultrarelativistic, and their thermal spectrum is exponential in $p_{t}$ : being boosted by flow it remains exponential with a "blue shifted" temperature $T^{*}=T \gamma_{t}$. For heavy particles the effect is quite different. In particular, an infinitely heavy particle has no thermal velocity and its thermal spectrum is $\sim \delta(\vec{p})$. If boosted by the flow, this delta functions just shifts its argument by $m v_{t}$, where $v_{t}$ is the flow velocity. The real nucleons or deuterons are in between of the two limits.

The findings of these two papers were however completely different. BEVALAC spectra discussed by Siemens and Rasmussen [8], for heavy ions at $E \sim 1 G e V * A$, indeed have shown the expected difference for pion and proton. They were well fitted with two parameters, the freezeout temperature $T_{f} \sim 30 \mathrm{MeV}$ and the velocity of what they have called the "blast wave" $v \approx 0.3^{5}$. Conclusions of our paper [5] was negative: the $\pi, K . N$ spectra from $p p$ collisions showed a very good $m_{t}$-scaling

$$
\frac{d N}{d p_{t}^{2}} \sim \exp \left(-\frac{m_{t}}{T}\right), \quad m_{t}^{2}=p_{t}^{2}+m^{2}
$$

with the same universal slope, without any sign of transverse collective flow. So, we all had to wait for heavy ion collisions at similar energies ${ }^{6}$.

\footnotetext{
${ }^{4}$ In fact Landau has been motivated by the opposite behavior of the effective charge in QED and scalar theories, growing toward small distances, which was before the asymptotic freedom considered the only possibility.

${ }^{5}$ Long discussion of whether hydro is applicable to derive it had followed: we would not go into that now.

${ }^{6}$ Incidentally, let me remind that around 1980-1982 it looked quite possible that CERN ISR would be used for that purpose. Unfortunately CERN leadership of the time decided otherwise, and even destroyed that steady and reliable collider "to store LEP magnets in the building". The extra irony of that announcement was the fact that LEP magnets have low field and were made mostly of concrete, with small admixture of iron, so there was no problem of their storage.
} 
It did indeed happened as planned, only decades later, at SPS and at RHIC. Fig:1(a), known as "Nu Xu plot", compares the pp data just discussed (open circles) with slopes of heavy ion spectra: as one can see these slopes grows approximately linearly with particle mass.

The last $d$ point is especially convincing: if flow interpretation is wrong and different $m_{t}$ slopes in AA and pp collisions observed are due to something else (e.g. to "initial state" rescattering, as some authors suggested), the $d$ slope of the $m_{t}$ spectra would be a convolution of $2 N$ spectra. A simple back-on-the-envelope calculation proves that for two uncorrelated nucleons the slope for $d$ should simply be the same as for $N$. This however is contrary to experimental observations: in fact the $d$ slope is about twice larger than $p$ one. Only pre-existing correlation between momenta of the two nucleons can explain that, and its magnitude is exactly as reproduced by the flow.

Inverse Slope Parameters in SPS
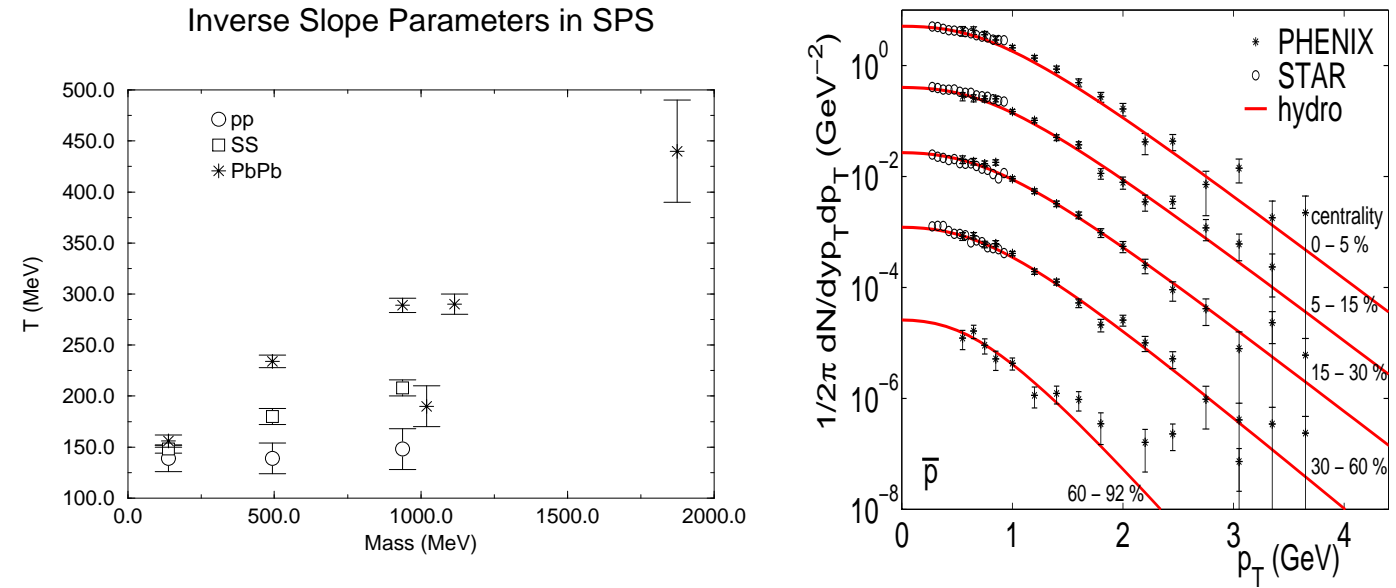

Figure 1: (a) Compilation of slopes of the $m_{t}$ spectra from pp collisions (ISR) (open circles), SS and $\mathrm{PbPb}$ collisions at SPS. (b) Comparison between STAR and PHENIX data for protons with hydro calculation by Kolb and Rapp [1] (which correctly incorporates chemical freezeout).

Let us now jump years ahead and show more modern version of the same argument. Fig \(b) shows recent RHIC data for the proton $p_{t}$ spectra, compared with hydro prediction. Note that no parameters other than fitted for pion spectra are present, and the agreement is very good, both in normalization and shape.

In Fig 2 we show a compilation of the results from RHIC (the STAR experiment) for pions, kaons and protons, fitted with hydro-inspired "blast wave" parameterization. It has two basic parameters the freezeout temperature $T_{k i n}$ and the mean flow velocity $\langle\beta>$. Different spectra are for different centrality classes, central at the top, the lowest curve in each case corresponds to $p p$ collisions. One can see that modification of the shapes of these spectra of all secondaries are nicely explained by the model. The parameter values, shown in the lower figures, show smooth variation with the centrality: $T_{k i n}$ decreases and the velocity increases. The reason for that was suggested by C.M.Hung and myself 9]: larger systems cool further. So, as the proverbial "rocket scientists", the most central collisions provide the strongest conversion of the internal energy into flow.

Note also that the temperature of chemical equilibration $T_{c h}$ seem to be completely independent of the centrality: the interpretation of it is that it is in fact the $Q C D$ critical temperature. For a reason which is not yet quite clear, most inelastic reactions seem to be rapidly quenched as soon as the matter is out of the QGP phase.

At RHIC not only a strong transverse flow with $v_{t} \sim .7$ was found, but (rather unexpectedly) hydro predictions with different slopes hold till rather large $p_{t} \sim 2 \mathrm{GeV}$. As a result, a crossing between pion and baryon spectra has been seen for the first time, so that there are as many nucleon as pions at and above such $p_{t}$, till $p_{t} \sim 5 \mathrm{GeV}$.

RHIC data have shown that strange quarks should flow together with the flow, as $\phi, \Omega$ made of strange quarks show similar behavior. One interesting questions to be addressed soon is whether the 

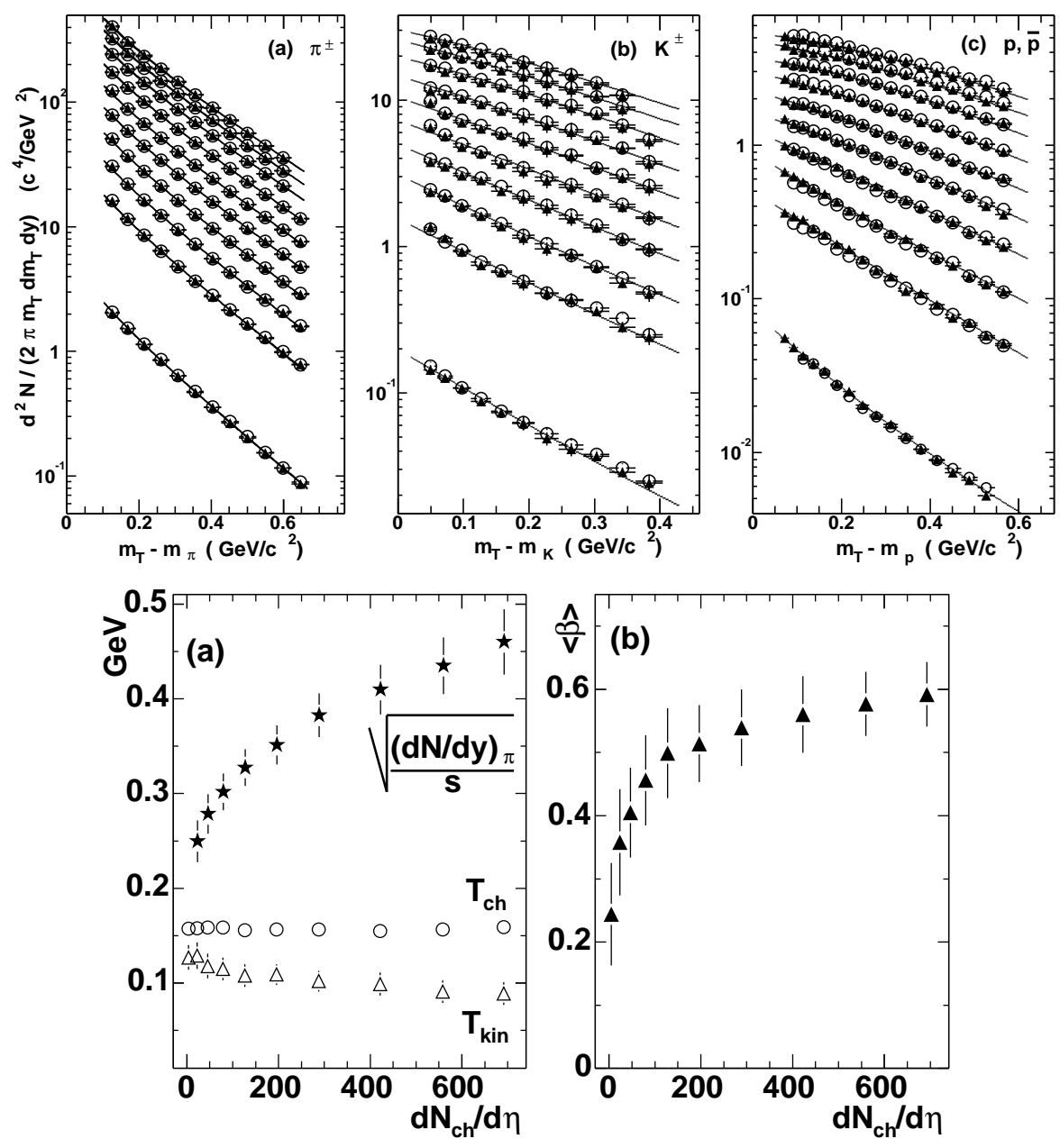

Figure 2: Pion, kaon and nucleon spectra from STAR collaboration (upper panels), together with the "blast model" fits. The values of the and freezeout temperatures are shown in (a) and the mean collective velocity in (b) part of the lower panel.

charmed quarks do (or do not) follow the flow as well: for that one should look at spectra of $D, J / \psi$ and other charmed particles.

\subsection{Qualitative effects of the QCD phase transition on flow}

We will not have time to discuss details of the hydrodynamics calculations, which reproduce these data. Let me only tell why RHIC collision energy is so special.

The QCD Equation of State (EOS) has a phase transition, so three respective stages of the "acceleration history" can be identified: (i) the matter is produced an the QGP phase $\left(e>e_{Q}\right)$, in which the matter accelerates rapidly because of substantial pressure $p \sim(1 / 3) \epsilon$; (ii) then the so called mixed phase follows $\left(e_{H}<e<e_{Q}\right)$, in which the matter is very soft, $p / \epsilon \ll 1$, and it basically free streams with zero acceleration; till finally (iii) the matter completely goes into a hadronic phase $\left(e<e_{H}\right)$, in which the relativistic pions produces substantial pressure and acceleration again. The effective EOS calculated along the appropriate adiabatic paths 9] from resonance gas plus QGP (from lattice) is shown in Fig 3 in the form $p / \epsilon$ versus $\epsilon$. The effect of the bag pressure on QGP is seen in this figure as a strong dip of this ratio, toward the so called softest point, the minimum of $p / \epsilon$. Since gradient of $\mathrm{p}$ is the driving force and $\epsilon$ is the mass to be moved, the acceleration of matter is proportional to this ratio. Its small value means that the mixed phase is much softer than both relativistic pion gas and high- $\mathrm{T}$ 
QGP on both sides of it. What this picture shows is that one can expect more robust hydro flow when the QGP produced is significantly more dense than at $T=T_{c}$, the "soft QGP". Heavy ion collisions at SPS energies were not high enough to have a substantial contribution of the first - QGP - stage, which happens for the first time at RHIC.

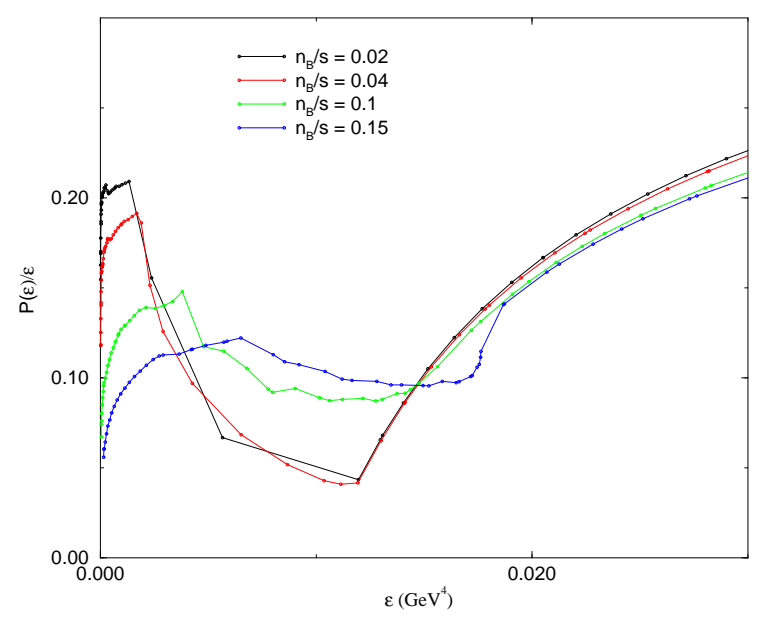

Figure 3: The dependence of the pressure-to-energy-density $p / \epsilon$ ratio on the energy density along the adiabatic paths with different baryon-to-entropy ratio. RHIC corresponds to the curve with the lowest baryon density, for which the contrast is the largest.

Compared to strong variation of the $p / \epsilon$, the observed dependence of the flow on the collision energy appears to be weak. While at low BEVALAC/SIS/AGS energies flow velocity $v_{t}$ steadily grows, for SPS and RHIC the difference is not that large. However, the relative contribution of stages (i) and (iii) have exchanged places, with QGP stage dominating flow values at RHIC.

Summarizing: Only with sufficiently high collision energy of RHIC we get to the regime in which the transverse expansion at the QGP stage is dominant. While large QGP pressure drives the initial transverse expansion, the "dark energy" (the bag constant or vacuum pressure) works against it.

\subsection{Elliptic Flow}

In non-central heavy ion collisions secondary particles emerge with an nontrivial "elliptic" flow pattern. The reason for it is that while "spectator" nucleons fly down the beam pipe, the produced fireball has an almond shape. If pressure develops in the system, its gradient is larger in the impact parameter direction (the x-direction) than in the longer $\mathrm{y}$-direction. Then, the excited matter expands preferentially in the $\mathrm{x}$-direction. This is quantified by $v_{i}$ harmonics defined $\mathrm{as}^{7}$

$$
\frac{d N}{d \phi}=\frac{v_{0}}{2 \pi}+\frac{v_{2}}{\pi} \cos (2 \phi)+\frac{v_{4}}{\pi} \cos (4 \phi)+\cdots
$$

which are measured experimentally ${ }^{8}$.

Each of $v_{i}$ is a function of centrality (the impact parameter $b$ ), rapidity $y$, transverse momentum $p_{t}$ and, last but not least, the particle type. All of those have been studied, but we have no time to go into these details. Just trust me, that hydro works nicely, for 99 percents of all secondaries or for

\footnotetext{
${ }^{7}$ We will not have time to discuss odd harmonics like $v_{1}$, which are nonzero away of mid-rapidity and measured at different collision energy including RHIC.

${ }^{8}$ Let me not go into an issue of the definition of the event plane. With several thousands of tracks seen in large detectors it is no longer a problem.
} 

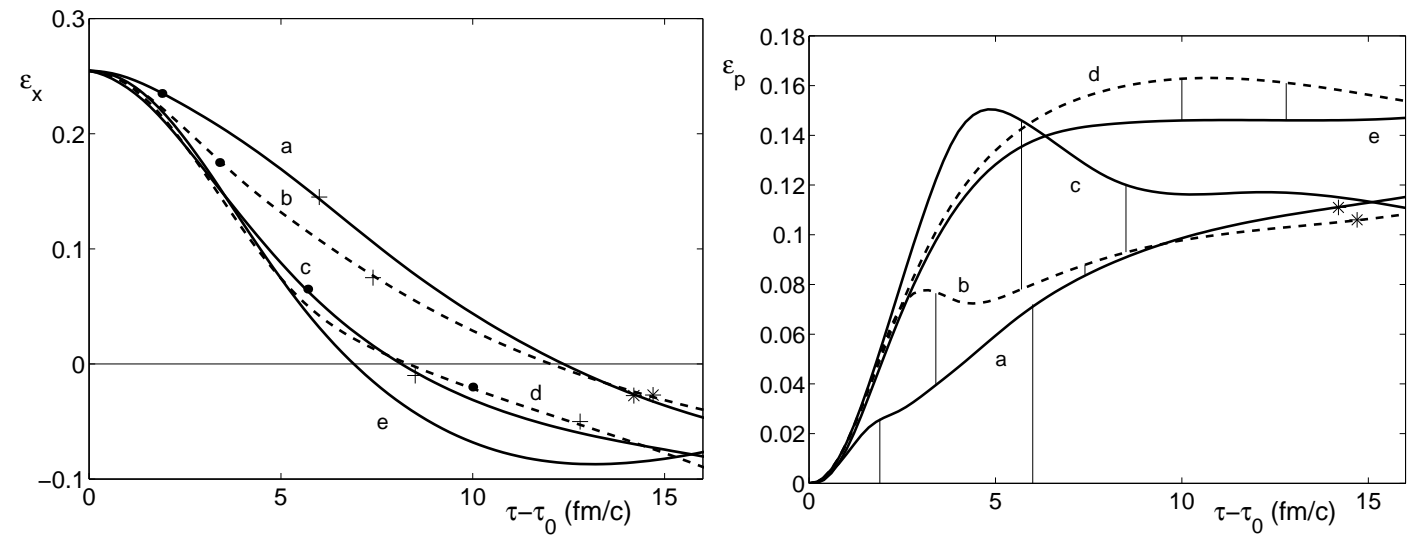

Figure 4: Time evolution of the spatial ellipticity $\epsilon_{x}$, the momentum anisotropy $\epsilon_{p}$, and the radial flow $\left\langle v_{\perp}\right\rangle$. The labels a, b, c and d denote systems with initial energy densities of 9, 25, 175 and 25000 $\mathrm{GeV} / \mathrm{fm}^{3}$, respectively, expanding under the influence of EOS Q. Curves e show the limiting behavior for EOS I as $e_{0} \rightarrow \infty$ (see text). In the second panel the two vertical lines below each of the curves a-d limit the time interval during which the fireball center is in the mixed phase. In the first panel the dots (crosses) indicate the time at which the center of the reaction zone passes from the QGP to the mixed phase (from the mixed to the HG phase). For curves a and $b$ the stars indicate the freeze-out point.; for curves c-e freeze-out happens outside the diagram.

$p_{t}<2 \mathrm{GeV}$. The data restrict the underlying EOS, the only theoretical hydro input, and the best description corresponds to the latent heat of about $0.8 \mathrm{GeV} / \mathrm{fm}^{3}$, which is in good agreement with the lattice predictions.

The important feature of elliptic flow is self-quenching. The time evolution of the spatial ellipticity $\epsilon_{x}$, the momentum anisotropy $\epsilon_{p}$, and the radial flow $\left\langle v_{\perp}\right\rangle$ are shown in Fig 4 . As the system expands, the eccentricity $\epsilon_{2}$ decreases. Since $\epsilon$ is the driving force behind the elliptic flow, the elliptic flow develops earlier than the radial one. This is why it is so important for understanding the EOS of the QGP.

The next Fig 5 makes use of one important fact: centrality dependence of $v_{2}$ is basically a response to the initial spatial anisotropy of the system, quantified by the parameter

$$
\epsilon \equiv \frac{\left\langle y^{2}-x^{2}\right\rangle}{\left\langle y^{2}+x^{2}\right\rangle},
$$

and so plotting $v_{2} / \epsilon$ one basically eliminates a geometric aspect of the problem and finds all points at some universal curve, see Fig 5 (a).

The main message of this figure is that $v_{2}$ at RHIC grows further with particle multiplicity. It has been theoretically predicted already at QM99, before RHIC was even completed. The parts $(\mathrm{b}, \mathrm{c})$ of the figure shows how the $v_{2}$ magnitude was expected to depend on collision energy ${ }^{9}$, from Teaney et al [10].

\subsection{The limits to ideal hydro}

It is not easy to reproduce hydro results using transport models. In fact it is only possible if the quasiparticle rescattering is increased by big factor relative to the pQCD expectations. The following Fig 6 from the kinetic studies by Gyulassy and Molnar [12 shows how the measured effect (boxes) can be reached while the matter opacity (= density times the cross section) grows. The smallest value on the plot roughly corresponds to gg scattering in pQCD, and one can see that it leads to no elliptic flow (or other collective effects) whatsoever.

\footnotetext{
${ }^{9}$ Other authors such as Ollitraught and Heinz et al have used fixed freezeout and predicted energy-independent $v_{2}$.
} 

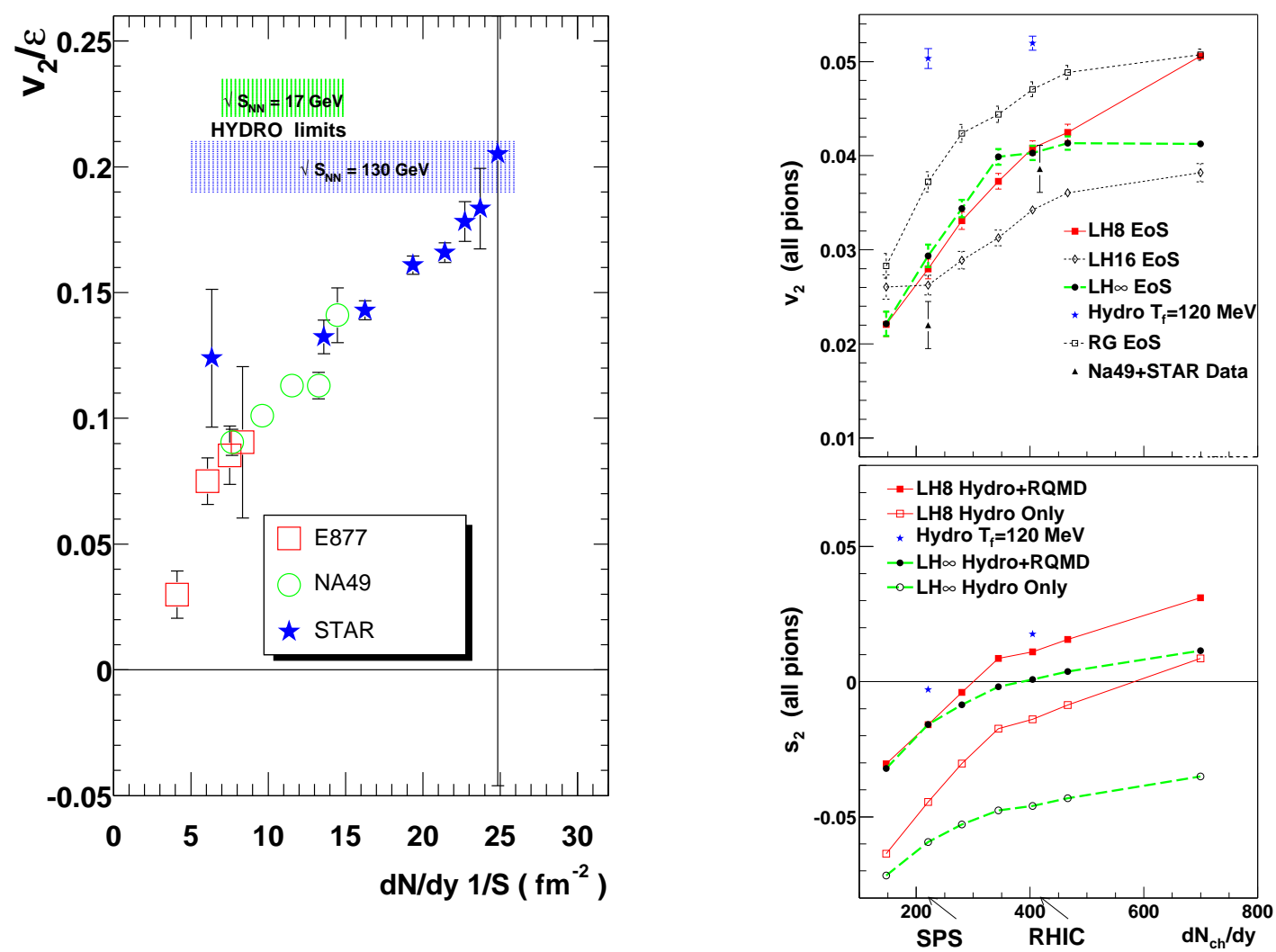

Figure 5: (a) The compilation of elliptic flow (the ratio of $v_{2} / s_{2}$ ) dependence on collision energy (represented by the particle multiplicity). (b,c) Elliptic flow predicted by hydro calculation by Teaney et al for different EoS. The curve with the latent heat $(\mathrm{LH})=800 \mathrm{MeV} / \mathrm{fm}^{3}$ is the closest to the lattice EoS, and it is also the best fit to all flow data at SPS and RHIC

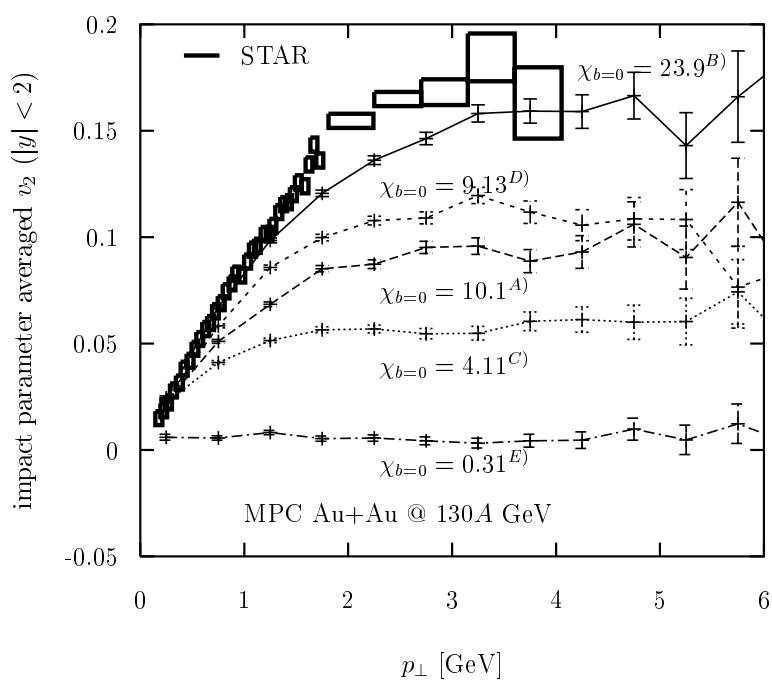

Figure 6: Impact parameter averaged gluon elliptic flow as a function of $p_{\perp}$ for $\mathrm{Au}+\mathrm{Au}$ at $\sqrt{s}=130 \mathrm{~A} \mathrm{GeV}$ with transport opacities $\chi_{b=0}=0.31,4.11,9.13,10.1$ and 23.9 for $b=0$. Identical to the charged hadron elliptic flow if the gluons are hadronized via local parton-hadron duality. 
The conceptual basis of the hydrodynamics ${ }^{10}$ is very simple: it is just a set of local conservation laws for the stress tensor $\left(T^{\mu \nu}\right)$ and for the conserved currents $\left(J_{i}^{\mu}\right)$,

$$
\begin{aligned}
\partial_{\mu} T^{\mu \nu} & =0 \\
\partial_{\mu} J_{i}^{\mu} & =0
\end{aligned}
$$

In equilibrium, $T^{\mu \nu}$ and $J_{i}^{\mu}$ are related to the bulk properties of the fluid by the relations,

$$
\begin{aligned}
T^{\mu \nu} & =(\epsilon+p) u^{\mu} u^{\nu}-p g^{\mu \nu} \\
J_{i}^{\mu} & =n_{i} u^{\mu}
\end{aligned}
$$

Here $\epsilon$ is the energy density, $p$ is the pressure, $n_{i}$ is the number density of the corresponding current, and $u^{\mu}=\gamma(1, \mathbf{v})$ is the proper velocity of the fluid. In strong interactions, the conserved currents are isospin $\left(J_{I}^{\mu}\right)$, strangeness $\left(J_{S}^{\mu}\right)$, and baryon number $\left(J_{B}^{\mu}\right)$. For the hydrodynamic evolution, isospin symmetry is assumed and the net strangeness is set to zero; therefore only the baryon current $J_{B}$ is considered below. These equations are valid if the microscopic length (such as mean free paths of constituents $l$ ) is small compared to the size of the system considered ${ }^{11} \mathrm{~L}$. Inclusion of dissipative effects, to the first order in $l / L$, is possible. Of course, the modified stress tensor and the currents no longer conserve the entropy, which is monotonously increased during the evolution.

Dissipative corrections to the stress tensor and the current can be written as follows

$$
\begin{gathered}
\delta T_{\mu \nu}=\eta\left(\nabla_{\mu} u_{\nu}+\nabla_{\nu} u_{\mu}-\frac{2}{3} \Delta_{\mu \nu} \nabla_{\rho} u_{\rho}\right)+\xi\left(\Delta_{\mu \nu} \nabla_{\rho} u_{\rho}\right) \\
\delta J_{\mu}=k\left(\frac{\eta T}{\epsilon+p}\right)^{2} \nabla_{\mu}\left(\mu_{B} / T\right)
\end{gathered}
$$

where the three coefficients $\eta, \xi, k$ are called the shear and the bulk viscosities and the heat conductivity, respectively. In this equation the following projection operator onto the matter rest frame was used:

$$
\nabla_{\mu} \equiv \Delta_{\mu \nu} \partial_{\nu}, \Delta_{\mu \nu} \equiv g_{\mu \nu}-u_{\mu} u_{\nu}
$$

Two general comments may be made in connection with these definitions. First, for matter with vacuum quantum numbers, characterized by the temperature only, one cannot even introduce the notion of heat conductivity. Second, bulk viscosity effects are very elusive, in particular, they vanish both in ultrarelativistic and nonrelativistic gas. So, the main non-equilibrium effect is connected with shear viscosity. Since, as we will show below, the late-time flow approaches radially symmetric Hubble flow without shear, the viscosity effect has a curious self-quenching property.

It is further useful to relate the magnitude of the viscosity coefficient $\eta$ to a more physical observable. As such one can use the sound attenuation length. If a sound wave have frequency $\omega$ and the wave vector $\mathbf{q}$, its dispersion law (the pole position) is

$$
\omega=c_{s} q-\frac{i}{2} \mathbf{q}^{2} \Gamma_{s}, \quad \Gamma_{s} \equiv \frac{4}{3} \frac{\eta}{\epsilon+p}
$$

In order to show how such parameter appears in hydro equations, let me give an example of the $1+1 \mathrm{~d}$ boost-invariant solution [7, corresponding to the velocity $u_{\mu}=(t, 0,0, z) / \tau$ where $\tau^{2}=t^{2}-z^{2}$ is the

\footnotetext{
${ }^{10}$ Although often hydrodynamics is treated as a consequence of kinetic equations, these two approaches have a different applicability ranges. Indeed, the stronger the interaction the better hydro works. The latter approach, on the contrary, was never formulated but for weakly interacting systems.

${ }^{11}$ Note that this is the same condition as for other macroscopic approaches, e.g. thermodynamics: however historically the hydrodynamical models met much more resistance than say statistical models.
} 
proper time. In this 1-d-Hubble regime there is no longitudinal acceleration at all: all volume elements are expanded linearly with time and move along straight lines from the collision point. Exactly as in the Big Bang, for each "observer" ( the volume element ) the picture is just the same, with the pressure from the left compensated by that from the right. The history is also the same for all volume elements, if it is expressed in its own proper time $\tau$. Thus the entropy conservation becomes the following (ordinary) differential equation in proper time $\tau$

$$
\frac{d s(\tau)}{d \tau}+\frac{s}{\tau}=0
$$

which has the obvious solution $s=\frac{\text { const }}{\tau}$.

Including first dissipative terms into our equations one has instead

$$
\frac{1}{\epsilon+p} \frac{d \epsilon}{d \tau}=\frac{1}{s} \frac{d s}{d \tau}=-\frac{1}{\tau}\left(1-\frac{(4 / 3) \eta+\xi}{(\epsilon+p) \tau}\right)
$$

Note that ignoring $\xi$ one finds in the r.h.s. exactly the combination which also appears in the sound attenuation, so the correction to ideal case is $\left(1-\Gamma_{s} / \tau\right)$. Thus the length $\Gamma_{s}$ directly tells us the magnitude of the dissipative corrections. Since the correction is negative, it reduces the rate of the entropy decrease with time. Another way to say that, is that the total positive sign shows that some amount of entropy is generated.

In order to understand qualitatively what viscous term would do it is convenient to use the simplest analytic example, Bjorken boost independent flow, and calculate what is added to the ideal isotropic pressure terms in the stress tensor. The result is that in longitudinal $z$ and transverse directions it is very different

$$
p_{z} \rightarrow p-\frac{4}{3} \frac{\eta}{\tau} \quad p_{\perp} \rightarrow p+\frac{2}{3} \frac{\eta}{\tau}
$$

Viscosity works against the longitudinal pressure, this is understandable: but why it helps the transverse one? The reason is particle distribution over momenta is deformed, in longitudinal direction it has less particles with large $p_{z}$ than the thermal one because those "jump" to other cells. But then distribution over $p_{\perp}$ should be somewhat wider, to keep mean chaotic energy the same. (Note that the sum of all 3 pressures is unchanged.)

Let us now discuss what is the value of QGP viscosity, following Teaney [13], who shown that one can also determine the viscous correction to particle distribution

$$
f=f_{o}\left(1+\frac{\eta}{s T^{3}} p^{\alpha} p^{\beta}\left\langle\nabla_{\alpha} u_{\beta}\right\rangle\right)
$$

where the gas is assumed to be a Boltzmann gas (otherwise $f_{o}\left(1 \pm f_{o}\right)$ is needed for Bose/Fermi ones). The tensorial structure makes is clear that any deviations are expected to be quadratic in momenta.

Such deviations from hydro predictions are indeed seen in spectra. In Fig 7 we show it for the elliptic flow parameter $v_{2}$. Since its value is determined at sufficiently early times - about $3 \mathrm{fm} / \mathrm{c}$ - the deviation should correspond to the QGP phase. The results for different $\Gamma_{s} / \tau$ shown in Fig 7 deviate from ideal hydro curve at $p_{\perp} \approx 1.6 \mathrm{GeV}$ which indicates $\Gamma_{s} / \tau \sim 0.05$ or so. Substituting here the relevant time $\tau \sim 3 \mathrm{fm} / \mathrm{c}$ we get $\Gamma_{s} \sim .15 \mathrm{fm}$. Strong coupling result for typical $T \sim 200 \mathrm{MeV}$ at the time gives $\Gamma_{s} \sim 0.1 \mathrm{fm}$, while weak coupling one would predict much larger value $\Gamma_{s} \sim 2 \mathrm{fm}$ or so. About the same value appears from a gluon cascade with the enhance cross section by Molnar and Gyulassy 12 we mentioned above. 


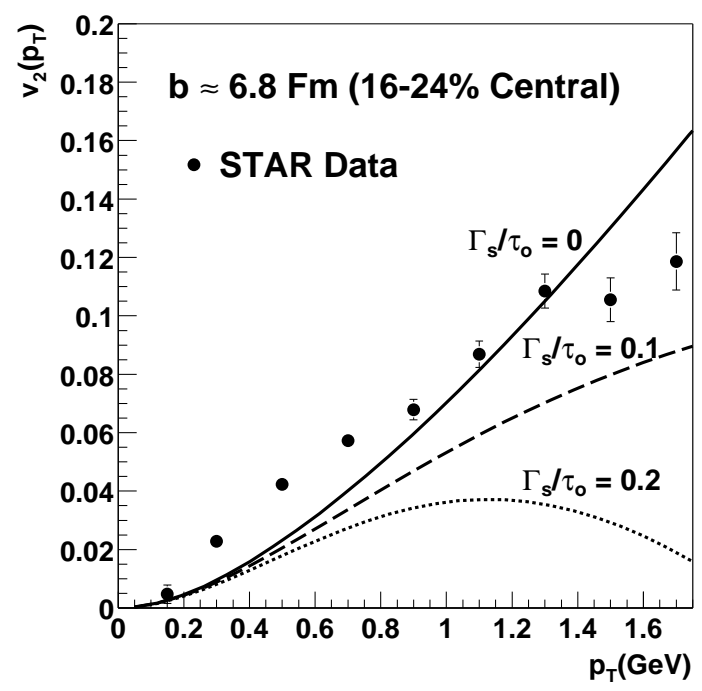

Figure 7: Elliptic flow $v_{2}$ as a function of $p_{T}$ for different values of $\Gamma_{s} / \tau_{o}$. The data points are four particle cumulants data from the STAR collaboration. Only statistical errors are shown.

\subsection{The "Little Bang" versus the Big Bang}

Completing the chapter on heavy ion collisions, let me make a more general comments emphasizing multiple amusing analogies between the heavy ion physics and the Big Bang cosmology.

First of all, the same hot/dense hadronic matter, which heavy ion physics is trying to produce in the laboratory, has been present at an appropriate time (microseconds) after Bing Bang. More specifically, in both cases the matter goes through the QCD phase transition, from the Quark-Gluon Plasma we discussed in the preceding chapter to the hadronic phase. On the phase diagram the Big Bang path proceeds along the $\mathrm{T}$ axis downward, since the baryonic density in it is tiny, $n_{B} / T^{3}<10^{-9}$. For heavy ion collisions the baryonic charge is small but still important. experiments.

The fireball created in the heavy ion collision of course explodes, as its high pressure cannot be contained: this is the Little Bang we referred to in the title of this section. The Big Bang is a cosmological explosion, which proceed against the pull of gravity. So, the Little Bang is a laboratory simulation of its particular stage. So, the first obvious similarities between the "Little Bangs" and cosmological "Big Bang" is that both are violent explosions.

In both cases the entropy is mysteriously produced at some early stage, and is approximately conserved later.

Expansion of the created hadronic fireball approximately follows the same Hubble law as its bigger relative, $v(r)=H r$ with $H(t)$ being some time-dependent parameter, although the Little Bang has rather anisotropic (tensorial) H. However, by the end of the expansion the anisotropy is nearly absent and local expansion at freezeout at RHIC is nearly Hubble-like.

The final velocities of collective motion in the Little Bang are measured in spectra of secondaries: the transverse velocities now are believed to be reasonably well known, and are not small, reaching about 0.7c at RHIC. For the Big Bang there is no end and it proceeds till today: the current value of the Hubble constant, $H\left(t_{\text {now }}\right)$ is, after significant controversies for years, believed to be reasonably well measured.

However already the next important question one would obviously ask, how exactly such expansion rates are achieved remains a matter of hot debates in both cases. The observed velocity of matter expansion at freezeout of the collisions or in Big Bang today is determined by the earlier acceleration, or the Equation of State (EoS) of the matter. 
For the Big Bang the EoS include gravity of all matter forms, including the still mysterious dark matter, as well as the (really shocking) dark energy (or the cosmological constant). Recent experimental observations of Supernovae in very distant Galaxies have concluded that the Bing Bang is accelerating at this time due to it.

Amusingly, the Little Bang has to deal with the cosmological constant as well. Of course, gravity is not important here, but going from QGP to hadronic matter one has to think about a definition of the zero energy density and pressure, or the so called bag constant between the two phases. As we will see, the cosmological term exists in this case, but it has the opposite sign and tries to decelerate expansion of QGP. The EoS is thus effectively very soft near the QCD phase transition: respectively the magnitude of the flow observed at AGS/SPS energies is not that large. With RHIC we had the first chance to go well beyond into the QGP domain, with harder EoS $p \approx \epsilon / 3$ and more robust expansion.

In a Little Bangs the observed hadrons (like microwave cosmic photons) are seen at the moment of their last interaction, or as we call it technical, at their freeze-out stage. In order to look deeper, one uses rare hadrons particles with smaller cross sections, such as $\Omega^{-}$hyperons, which decouple earlier, or even penetrating probes [1] leptons or photons which penetrate through the whole system. We return to their discussion in the next chapter.

The next comparison I would like to make here deals with the issue of fluctuations. Very impressive measurements of the microwave background anisotropy made in the last decade have taught us a lot about cosmological parameters. First the dipole component was found - motion of the Solar system relative to the microwave heat bath, and then a very small $\left(\delta T / T \sim 10^{-5}\right)$ chaotic fluctuations of $\mathrm{T}$ originated from plasma oscillations at the photon freeze-out. It has been possible lately to measure some interesting structures in fluctuations of cosmic microwave background, with angular momenta $l \sim 200$. The theoretical predictions for these were available since it is related to primordial plasma-togas transition at temperature $T \sim 1 / 3 \mathrm{eV}$. Primordial fluctuations of sufficiently long wavelength are attenuated by the gravitational instability, till they reach the stabilization moment $\tau_{\text {stabilization }}$ when the instability is changed to a regime called Sakharov acoustic oscillations. Hydrodynamics tells us that fluctuations disperse during this period with the (current) speed of sound, reaching the so called sound horizon scale

$$
r_{s . h .}=\int_{\tau_{\text {stabilization }}}^{\tau_{\text {observation }}} c_{s}(t) d t
$$

This scale is the physical size of spots observed, and correspond to a peak at $l \approx 220$ recently observed.

In heavy ion collisions similar studies are in its infancy. There exist of course the global ellipticity of the event, related to elliptic flow and nonzero impact parameters: we will discuss it below in detail in section 2.4. We do not yet see reliable signals for either mean values of higher harmonics, or their fluctuations. Nevertheless, since in the mixed phase the transition of high density QGP phase into low density hadronic gas also should be characterized by some instabilities, the sound horizon is also a useful concept to define the observed spectrum of the fluctuations ${ }^{12}$ Although we do not yet have any observations of higher harmonics and their fluctuations, I think there is a chance to see eventually "frozen plasma oscillations" in this case as well. After all, RHIC produced millions of events, while The Big Bang remains the only one! At the end of the day, the amount of information taken about the Little and Big Bangs are comparable ${ }^{13}$.

\section{Transport properties of the QGP}

The perturbative approach to transport is well summarized by P.Arnold et al [14]. The high temperature shear viscosity in a gauge theory with a simple gauge group (either Abelian or non-Abelian) has the

\footnotetext{
${ }^{12}$ The paper on that subject I am working on is in progress at this point.

${ }^{13}$ In the final analysis, both are determined by number of pixels in the detectors and computer storage.
} 


\begin{tabular}{cc}
$n_{\mathrm{f}}$ & $\eta \times\left(g^{4} / T^{3}\right) \ln g^{-1}$ \\
\hline 0 & 27.126 \\
1 & 60.808 \\
2 & 86.473 \\
3 & 106.664 \\
4 & 122.958 \\
5 & 136.380 \\
6 & 147.627
\end{tabular}

Table 1: Leading-log shear viscosity as a function of the number of (fundamental representation) fermion flavors with $m \ll T$, for gauge group $S U(3)$.

leading-log form

$$
\eta=\kappa \frac{T^{3}}{g^{4} \ln g^{-1}},
$$

where $g$ is the gauge coupling, which is presumed to be small. For the case of $S U(3)$ gauge theory the leading-log shear viscosity coefficient $\eta$ for various numbers of light $m<<T$ fermion species are shown in the Table 1. One may conclude from it that $\eta / s$ should be very large compared to 1.

We will discuss the $\mathcal{N}=4$ supersymmetric Yang-Mills theory below, but let me jump ahead and mention here the strong coupling result for the viscosity by Policastro, Son and Starinets [15]. Their main result for shear viscosity is

$$
\eta=\frac{\pi}{8} N_{c}^{2} T^{3}
$$

which, after it is combined with the results for thermodynamics [24], shows that the dimensionalless viscosity-to-entropy ratio is small, specifically

$$
\frac{\eta}{s}=\frac{1}{4 \pi}
$$

They also found other evidences that hydrodynamics is a good theory in the strong coupling limit.

In subsequent works [16] more examples of Maldacena-like correspondence were studied, and this particular ratio was found to be the same for all cases. These authors conjectured that it is in fact the lowest possible value of this ratio, representing the most perfect liquid possible.

\subsection{Rethinking the QGP at RHIC, $T_{c}<T<2 T_{c}$}

The earliest suggested QGP signal was a disappearance of familiar hadronic peaks - $\rho, \omega, \phi$ mesons in the dilepton spectra [1]. Moreover, even small-size deeply-bound $\bar{c} c$ states, $\eta_{c}, J / \psi$, were expected to melt at $T \approx T_{c}[17,18]$.

However, it turns out that in wide region $T<4 T_{c}$ the interaction is strong enough, causing bound states of quasiparticles. More specifically, those are limited by the zero binding lines on the phase diagram (see Fig 8 (a)), introduced by I.Zahed and myself in [2] .

Discussion of particular hadronic states in QGP have been done many times before, see e.g. application of the sum rules or instantons. There are also important lattice results. It has been shown [19] that (time-direction) correlators at $T>T_{c}$ display significant deviations from free behavior, in quantitative agreement with predictions in [21. Their analysis (by the minimal entropy method) have suggested existence of light quark resonances above $T_{c}$. Most definite are recent studies [19] which found (contrary to earlier expectations) that the lowest charmonium states remain bound up to $T<T_{\bar{c} c} \approx(1.6-2) T_{c}$. 

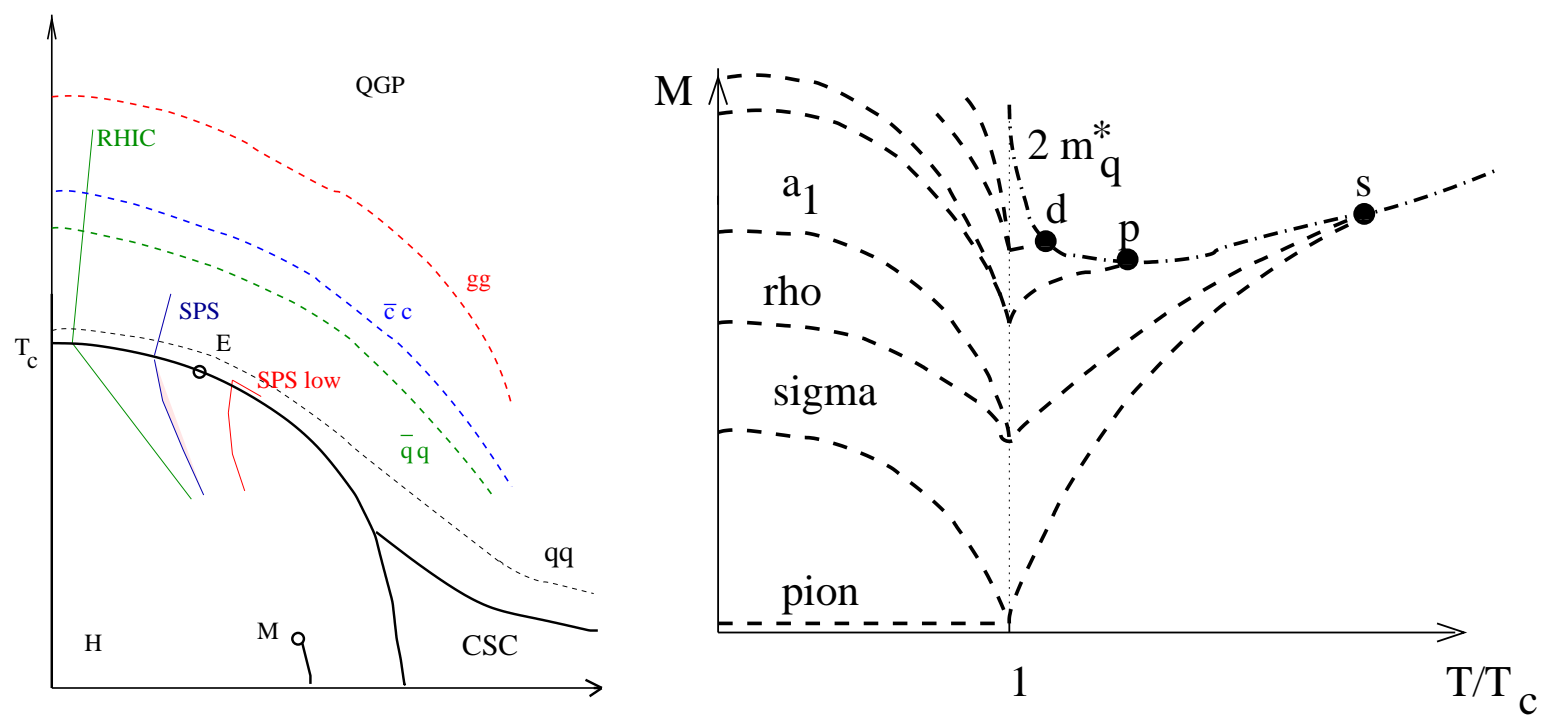

Figure 8: Schematic position of several zero binding lines on the QCD phase diagram (a) and of specific hadronic masses on temperature $T$ (b). In the latter the dash-dotted line shows twice the (chiral) effective mass of a quark. Black dots marked $s, p, d$ correspond to the points where the binding vanishes for states with orbital momentum $l=0,1,2 \ldots$.

The bound states of $\bar{q} q$ can only be colorless mesons (the octet channel is repulsive), but in QGP there can be colored bound states. Quite famous are quark Cooper pairs $q q$ which drive the color superconductivity at sufficiently high density and low $T$ : but pairs themselves should exist outside this region as well. Gluons can form a number of states with attraction, and there can also be $g q$ hybrids. A generic reason why we think all of them exist is that at $T$ close to $T_{c}$ all quasiparticles are very heavy.

Starting with quarks, let us briefly discuss their quantum numbers. Ignoring current quark masses and instanton effects, we note than in QGP the chiralities (L,R) are good quantum numbers. Furthermore, there are two different modes of quarks depending on whether helicity is the same as chirality or not (these states are called "particle" and "plasmino", respectively). All in all there are $4 N_{f}^{2}$ states, connected by continuity to the pseudoscalar, scalar, vector and axial vector nonets in the vacuum (see Fig $8(b))$.

Gluons in QGP have not 2 but 3 polarizations, with a longitudinal "plasmon" mode. Furthermore, for $g g$ one can have not only color singlet (a continuation of the vacuum glueballs) but also octet binary composites. The exotic states $q q, q g$ or octet $g g$ have smaller Casimirs and need stronger couplings, so they are melted first.

Our main idea is that a sequence of loosely bound states (indicated by black dots in Fig 8 (b)) is of crucial importance for quasiparticle rescattering, since there its cross section is strongly enhanced. Huge cross sections induced by low-lying resonances are well known throughout all parts of physics. The Breit-Wigner cross section (modulo the obvious spin factors depending on the channel) is

$$
\sigma(k) \sim \frac{4 \pi}{k^{2}} \frac{\Gamma_{i}^{2} / 4}{\left(E-E_{r}\right)^{2}+\Gamma_{t}^{2} / 4}
$$

For $E-E_{r} \approx 0$ the in- and total widths approximately cancel: the resulting "unitarity limited" scattering is determined by the quasiparticle wavelengths which can be very large. We conjecture that this phenomenon significantly contribute to viscosity reduction.

The issue of $\bar{c} c$ binding can be addressed using the usual non-relativistic Schrodinger equation, which for standard radial wave function $\chi(r)=\psi / r$ has the usual form, with the reduced mass $m=$ $m_{c} / 2$ and an effective potential including T-induced screening of the charge [1, which we will write 
in a traditional Debye form $V=-\left(4 \alpha_{s}(r) / 3 r\right) \exp \left(-M_{D} r\right)$. We have chosen the (T-dependent) inverse screening mass to be our unit of length, so that the equation to be solved reads

$$
\frac{d^{2} \chi}{d x^{2}}+\left(\kappa^{2}+\frac{4 m_{c}}{3 M_{D}} \frac{\alpha_{s}(x)}{x} e^{-x}\right) \chi=0
$$

with $\kappa^{2}=m_{c} E / M_{D}^{2}$. Furthermore, at a zero binding point $\kappa=0$. So, if the coupling constant $\alpha_{s}$ would not run and be just a constant, all pertinent parameters appear in a single combination. Solving the equation, one finds the zero binding condition to be

$$
\frac{4 m_{c}}{3 M_{D}} \alpha_{s}=1.68
$$

For example, using $4 / 3 \alpha_{s}=0.471$ and $m_{c}=1.32 \mathrm{GeV}$, as Karsch et al [18] did long ago, one finds a restriction on the screening mass $M_{D}<M_{D}^{\text {crit }}=0.37 \mathrm{GeV}$. Lattice measurements of the screening masses for the near-critical QGP [20] found that (not very close to $\left.T_{c}\right) M_{D} / T \approx(2.25 \pm .25)$ in a relevant range of $T$. If so, the condition (20) is satisfied marginally if at all, and these authors concluded that charmonium s-wave states $\eta_{c}, J / \psi$ cannot exist inside the QGP phase.

A loophole in this argument is the assumption that the gauge coupling is a constant, with the same value as in the in-vacuum charmonium potential. However in-vacuum potential includes large confining linear term, absent at $T>T_{c}$. The true form of $V_{\text {eff }}(r)$ remains unknown. At $T>T_{c}$ nothing prevents the QCD coupling from running at larger distances to larger values until it is stopped at the screening mass scale. Very close to the critical point, where the screening mass is very small, the coupling can reach $\alpha_{s} \sim 1$ and presumably be limited by some nonperturbative phenomena.

we use a simple model-dependent potential, in which the charge continues to run till it is limited by $\alpha_{s}<1$. Such potential with the running coupling does indeed provide a more liberal condition for charmonium binding, which is $M_{D}<0.62 \mathrm{GeV}$. This translates into charmonium zero binding point at

$$
T<T_{\bar{c} c} \approx 1.6 T_{c}
$$

which agrees well with recent lattice measurements [19]: we thus conclude that our model-dependent potential has passed its first test.

The binding of light $\bar{q} q$ states. Chiral symmetry for massless quarks excludes the usual mass from being developed, and $L, R$-handed quarks propagate independently. Nevertheless, propagating quasiparticles if the QGP have dispersion curves with the nonzero "chiral" or "thermal" mass, defined as the energy of the mode at zero momentum $M_{q}=\omega(\vec{p}=0)$. Perturbatively it is $M_{q}=g T / \sqrt{6}$ to the lowest order, the same for both fermionic modes: i. with the same chirality and helicity the dispersion curve at small $p$ is $\omega=M_{q}+p / 3+p^{2} / 3 M_{q}+\ldots$; ii. with the opposite chirality and helicity the mode is often called a "plasmino", its dispersion curve has a shallow minimum at $p=0.17 g T$ with the energy $E_{\text {min }}=0.38 g T$ slightly below $M_{q}$. For a general analysis of these modes see [23].

Lattice data on the quasiparticle dispersion curves are rather sketchy, obtained in a Coulomb gauge [22]. They can be described by $\omega^{2}=p^{2}+M^{2}$, with the following values $\left(\right.$ at $\left.T=1.5 T_{c}\right)$

$$
\frac{m_{q}}{T}=3.9 \pm 0.2 \quad \frac{m_{g}}{T}=3.4 \pm 0.3
$$

Note that at such $T$ (which is not very close to $T_{c}$ ) the quark and gluon masses are still quite large, while their ratio is very different from the weak coupling prediction $1 / \sqrt{6}$. At $T \sim 3 T_{c}$ and higher they are somewhat reduced toward the perturbative values, which however are not reached till presumably very high $T$.

The effective equation of motion suitable for discussion of the bound state problem can be obtained by standard substitution of the covariant derivatives in the place of momentum and frequency, provided 
the dispersion law is known. So if the dispersion curve can be parameterized as $\omega=M_{q}+p^{2} / 2 M^{\prime}+.$. , it has the form of the nonrelativistic Schrodinger equation, in general with two different constants $M, M^{\prime}$. Both for weak coupling and lattice data, such approximation seem to be accurate withing several percents.

Addressing the issue of binding, we first note that if all effective masses grow linearly with $T$, including the screening mass, the explicit $T$ dependence drops out of (20) to the exception of the logarithmic dependence in $T$ left out in $\alpha_{s} \sim 1 / \ln \left(T / \Lambda_{Q C D}\right)$. This is why the region of "strongly coupled QGP" turns out to be relatively substantial. For a qualitative estimate, let us set the coupling to its maximum, $\alpha_{s}=1$. The combination of constants is $(4 \times 3.9 T) /(3 \times 2.25 T)=2.3$, larger than the critical value (20), so one should expect the occurrence of (strong) Coulomb bound states. Although the (plasmon) gluon modes are somewhat lighter than quarks in (22), their Coulomb interaction has a larger coefficient due to a different Casimir operator for the adjoint representation, 3 instead of 4/3. As a result the effective combination in the potential is $3 m_{g} \alpha_{s} / M_{D}$, which is about twice larger than for quarks, and thus the gluons are bound even stronger (modulo collisional broadening).

Solving the equations one can make a quantitative analysis, using the same potential as above. We found that the highest temperature $T$ at which light quark states are Coulomb bound is somehow lower than that of charmonium,

$$
T_{\bar{q} q} \approx 1.45 T_{c} \approx 250 \mathrm{MeV}
$$

while the s-wave $g g$ gluonium states remain bound till higher temperatures $T_{g g} \approx 4 T_{c}$ used in this paper as the upper limit on the QGP with bound states.

How reliable is our approach, based on screened effective potential? Obviously even the best inmatter potential does not include all many-body effects. However one may think that large size of states at near-zero binding provides additional stability, averaging out local perturbations. This is known to be true for large-size Cooper pairs in superconductors, or "excitons" in semiconductors and insulators. Depending on a number of parameters, including the density of excitons and temperature, this system exhibits various phases, ranging from an ideal gas of excitons to a liquid or plasma, or even a Bose-condensed gas. On its way from a gas to a liquid, clustering with 3- and 4-body states play an important role. Although one cannot directly relate these two problems (quarks and gluons have $N_{c}$ and $\left(N_{c}^{2}-1\right)$ colors respectively, while particles and holes have simply charges $\pm e$ ), one may think that in the QGP at $T \sim T_{c}$ some of these phenomena may well be there.

In a more recent paper, by Brown et al [3], the fate of the $\bar{q} q$ bound states is traced to $T \approx T_{c}$, where the Nambu-Goldstone and Wigner-Weyl modes meet. the binding of these states is accomplished by the combination of (i) the color Coulomb interaction, (ii) the relativistic effects, and (iii) the quasi-local interaction induced by the instanton-anti-instanton molecules. The spin-spin forces turned out to be small.

While near $T_{z b}$ all mesons are large-size nonrelativistic objects bound by Coulomb attraction, near $T_{c}$ they get much more tightly bound, with the $\sigma$ and $\pi$ masses approach zero (in the chiral limit). The wave function at the origin grows strongly with binding, and the near-local four-Fermi interactions induced by the instanton molecules play an increasingly more important role as the temperature moves downward toward $T_{c}$. 


\section{The $\mathcal{N}=4$ supersymmetric gauge field theory at strong cou- pling}

\subsection{The main results obtained via the Maldacena duality}

In QCD, as soon as the lowest states $\pi, \sigma$ hit zero mass, there is an instability leading to a phase transition into hadronic (confining and chirally asymmetric) state. This is however impossible for conformal gauge theories (CFTs), such as $\mathcal{N}=4$ supersymmetric gauge theory. In CFT the gauge coupling is allowed to become supercritical or even large $\lambda \equiv g^{2} N_{c} \gg 1$.

In order to compare it properly with the QCD we remind that on top of the $S U\left(N_{c}\right)$ gauge fields this theory has 4 gluino-like fermions and 6 scalars $^{14}$. For 3 colors this is 16 gluons, plus 64 fermionic d.o.f. (to be compared to $12 N_{f}$ with anti-fermions in QCD) plus 48 scalars.

Further modification of the background metrics 24] with a Schwartzschild (black hole) has allowed to consider $\mathcal{N}=4$ theory at finite temperature $T$. The metrics used in all calculations to be referred below is thus

$$
d s^{2}==\alpha^{\prime}\left[\frac{1}{\sqrt{G}}\left(-H d t^{2}+d \mathbf{x}_{\|}^{2}\right)+\sqrt{G}\left(\frac{1}{H} d \mathrm{U}^{2}+\mathrm{U}^{2} d \mathbf{\Omega}_{5}^{2}\right)\right]
$$

where $\alpha^{\prime}$ is the string tension, $t, \mathbf{x}_{\|}$are 4-d coordinate of our space-time, $\Omega_{5}$ is the 5 - $\mathrm{d}$ solid angle of the 5 -d sphere, $G \equiv \frac{g_{\text {eff }}^{2}}{\mathrm{U}^{4}}$

$$
H \equiv 1-\frac{\mathrm{U}_{0}^{4}}{\mathrm{U}^{4}} \quad \mathrm{U}_{0}^{4}=\frac{2^{7} \pi^{4}}{3} g_{\mathrm{eff}}^{4} \frac{\mu}{N^{2}} .
$$

where $g_{\text {eff }}^{2}=g^{2} N_{c}$ is the 't Hooft coupling. The parameter $\mu$ is interpreted as the free energy density on the near extremal D3-brane, hence, $\mu \approx\left(4 \pi^{2} / 45\right) N^{2} T^{4}$. So the metrics without the black hole, $T=0$ corresponds to $H=1$.

The thermodynamics of this is discussed extensively in [24] with the conclusion that in the strong coupling limit $\epsilon=3 p$ reaches $3 / 4$ of its Stephan-Boltzmann value. The corrections are $O\left(1 / \lambda^{3 / 2}\right)$. It is very instructive to compare weak and strong coupling results, which we do in Fig.9. The pressure, shown in Fig.(a), presumably show a monotonous decrease from 1 to $3 / 4$, but both weak and strong coupling results seem to be quite wrong for $\lambda=g^{2} N_{c}=1 . .5$.

The Maldacena's duality [25], known also as Anti-deSitter AdS/CFT correspondence, has opened a way to study this strong coupling limit using classical gravity. At finite $T$ it was recently actively discussed, for Debye screening [26, bulk thermodynamics 24] and kinetics [15]. Although the thermodynamical quantities are only modified by an overall factor of $3 / 4$ in comparison to the black-body limit, kinetics is changed dramatically.

In our second paper 4 related with bound states in QGP-like phases we show that in this regime the matter is made of very deeply bound binary composites, in which the supercritical Coulomb can be balanced by centrifugal force. a re-summation of a class of diagrams, in vacuum and at finite $T$. Specific towers of such bound states can be considered as a continuation of Fig 8 to the left, toward stronger and stronger coupling.

$N=4$ super-Yang-Mills (SYM) is the most famous example of a Conformal Field Theory (CFT) in 4 dimensions. This theory has zero beta function and a non-running coupling constant, which can be continuously changed from weak to strong. Unlike QED or QCD where for a critical coupling $\alpha \approx 1$ there is vacuum rearrangement, the CFT is believed to remain in the same Coulomb (plasma-like) phase for all couplings. Thus, it provides an interesting theoretical laboratory for understanding properties of

\footnotetext{
${ }^{14}$ This is needed by supersymmetries, to balance the number of degrees of freedom $\left(N_{c}^{2}-1\right) *(2-8+6)=0$, where we use minus for fermions.
} 


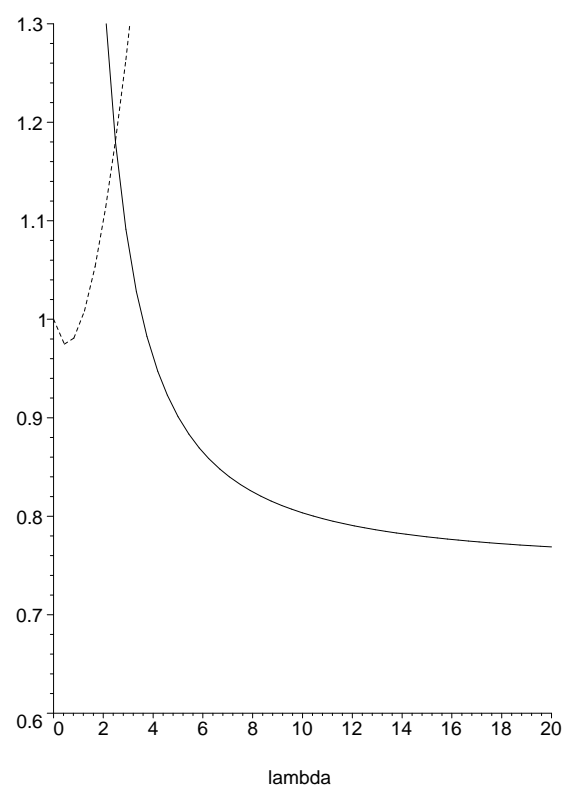

Figure 9: Comparison of the weak ( 5 terms) and strong (2 terms) coupling series, for the $\mathcal{N}=4$ theory. The plot shows the pressure $\mathrm{p}$ (in units of its to Stephan-Boltzmann value) versus the coupling $\lambda=g^{2} N_{c}$. At $\lambda=2-6$ both expansions seem to fail; but presumably the true line just smoothly interpolates. In QCD at $T \sim 1.5 T_{c}$ the effective coupling is at its maximum which corresponds to $\lambda \sim 10-20$, which seem to be firmly on the strong coupling side.

a strongly coupled Quark-Gluon Plasma (QGP) in QCD, which occurs at $T \approx T_{c}$ as discussed in our previous paper [2].

A key breakthrough in understanding the strong coupling regime was the AdS/CFT correspondence conjectured by Maldacena [25]. The conjecture has turned the intricacies of strong coupling gauge theories to a classical problem in gravity albeit in 10 dimensions. For instance, the static potential between a heavy quark and antiquark derived from the asymptotic of an elongated temporal Wilson loop, follows from a minimal surface (classical string) between the quarks stretched by gravity (metric of the AdS space) as depicted in Fig. 1a. The result is a modified Coulomb's law $(\lambda \gg 1)[25]$.

$$
V(L)=-\frac{4 \pi^{2}}{\Gamma(1 / 4)^{4}} \frac{\sqrt{\lambda}}{L}
$$

The numerical coefficient in the first bracket is 0.228 . The latter will be compared to the result from a diagrammatic re-summation below.

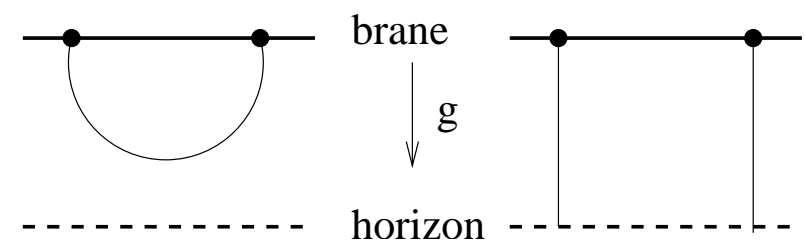

Figure 10: Two types of solutions describing the potential between two static charges (large dots) in the ordinary 4-d space (on the D3 brane). The string originating from them can either connect them (a) or not (b). In both cases the string is deflected by a background metric (the gravity force indicated by the arrow marked g) downward, along the 5-th coordinate. After the string touches the black hole horizon (b) a Debye screening of the interaction takes place. 
The case of the non-zero temperature is represented in the AdS space by the occurrence of a blackhole in the 5th dimension, whereby its Schwartzschild radius is identified with the inverse temperature. When the string between charges extends all the way to the black hole as shown in Fig. 1b, the heavy quark potential is totally screened for a Debye radius of order $1 / T$ 26. This is to be contrasted with $\sqrt{\lambda} T$ expected in the weak coupling limit for the electric modes, and $\lambda T$ for the magnetic modes.

The main puzzle related with all strong coupling results whether it is the free energy, the viscosity, or the resonance frequencies, is their independence on the coupling $\lambda$ in strong coupling. We recall that the interaction between the (quasi) particles such as (26) is proportional to $\sqrt{\lambda}$. In a naive picture of a quasiparticle plasma, familiar from the weak coupling limit, one would expect the interaction terms to show up in the free energy.

\subsection{Summing the ladder diagrams}

The main objective of our paper [4] was to explain these puzzles, but before that one should understand the dynamical picture behind the modified Coulomb law, and its Debye-screened form at finite $T$ and strong coupling. For that, one can identify in the gauge theory a set of diagrams whose re-summation can reproduce the parametric features of the above mentioned strong coupling results. As a result, we learn an important lesson: in the strong coupling regime even the static charges communicate with each other via high frequency gluons and scalars, propagating with an effective super-luminal velocity $v \approx \lambda^{1 / 4} \gg 1$.

The observation that potential-type diagrams in Feynman gauge reproduce the strong coupling regime has been made by Semenoff and collaborators [27], who have shown that the ladder re-summation works for the circular Wilson loop and qualitatively explains the modified Coulomb law at strong coupling and zero temperature.

Intuitively, the reason for a potential-like regime stems from the fact that for $\lambda \gg 1$ the time between subsequent exchange of quanta is very short. The cost of repulsive Coulomb energy becomes prohibitively large at strong coupling, forcing both charges to almost simultaneously change their colors, keeping them oriented in mutually the most attractive positions. These interactions are naturally ordered in time, justifying the use of ladder-type approximations.

Let me start by reminding the reader of the (Euclidean) derivation of the standard Coulomb's law between two attractive and abelian static charges. In the first quantized form in Feynman gauge one simply gets it from a 00-component of the photon (gluon) propagator

$$
V(L) \approx-\frac{\lambda}{4 \pi^{2}} \int_{-\infty}^{+\infty} \frac{d t}{t^{2}+L^{2}}
$$

where $t$ is the relative time separation between the two charges on their world lines. In the abelian case whether at strong or weak coupling, the interaction takes place at all time virtualities resulting into the standard instantaneous Coulomb interaction with $V(L) \approx-\lambda / L$. The non-abelian modified Coulomb's law (26) is seen to follow from the abelian Coulomb's law (27) whereby the relative time interval is much shorter and of order $L / \sqrt{\lambda} \rightarrow 0$.

We start by reminding the reader of some results established in [27] at zero temperature. At large $N_{c}$ (number of colors), the diagrams can be viewed as t'Hooft diagrams. An example of a ladder diagram is shown in Fig. 11 , where the rungs can be either gluons and scalars, as both are in the adjoint representation. The first lesson is that each rung contributes a factor $N_{c}$, which however only comes from planar diagrams. It means, that in contrast to the Abelian theory, the time ordering should be strictly enforced, $s_{1}>s_{2}>s_{3} \ldots$ and $t_{1}>t_{2}>t_{3} \ldots$, which will be important below.

In Fig. 11b we schematically depict the Bethe-Salpeter equation. The oval connected with the two Wilson lines by multiple gluon/scalar lines is the re-summed Bethe-Salpeter kernel $\Gamma(s, t)$, describing the evolution from time zero to times $s, t$ at two lines. It satisfies the following integral equation 


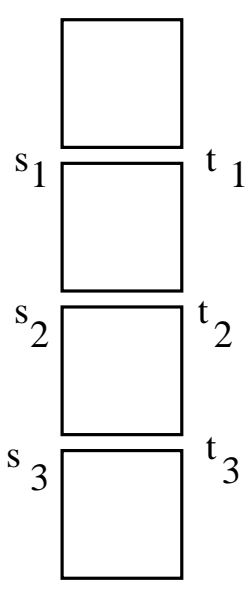

(a)

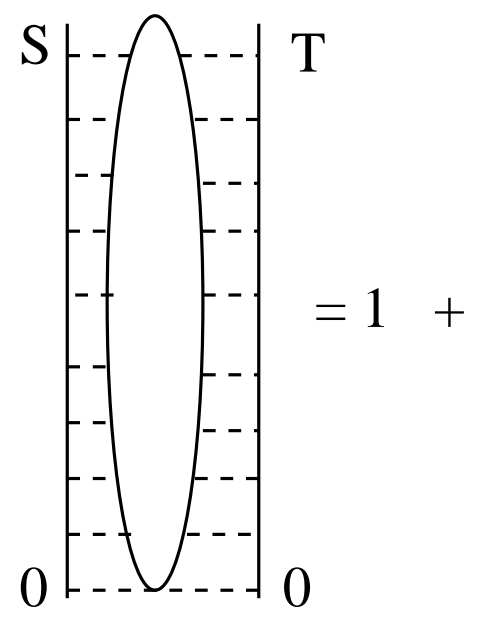

(b)

Figure 11: (a) The color structure of ladder diagrams in the large- $N_{c}$ limit: each square is a different color trace, bringing the factor $N_{c}$. The time goes vertically, and the planarity condition enforces strict time ordering, $s_{1}>s_{2}>s_{3} \ldots, t_{1}>t_{2}>t_{3} \ldots$ (b) Schematic representation of the Bethe-Salpeter equation (28) summing ladders.

$$
\Gamma(S, T)=1+\frac{\lambda}{4 \pi^{2}} \int_{0}^{S} d s \int_{0}^{T} d t \frac{1}{(s-t)^{2}+L^{2}} \Gamma(s, t)
$$

which provides the re-summation of all the ladder diagram. $L$ is the distance between two charges, and the first factor under the integral is the (Euclidean) propagator for one extra gluon/scalar added to the ladder. The kernel obviously satisfies the boundary condition $\Gamma(S, 0)=\Gamma(0, T)=1$. If the equation is solved, the ladder-generated potential follows from $V_{\text {lad }}(L)=-\lim _{T \rightarrow+\infty} \frac{1}{T} \Gamma(T, T)$,.

In weak coupling $\Gamma \approx 1$ and the integral on the rhs is easily taken, resulting in

$$
\Gamma(S, T) \approx 1+\frac{\lambda}{8 \pi} \frac{S+T}{L}
$$

which results into the standard Coulomb's law. Note that in this case the typical relative time difference between emission and absorption of a quantum $|t-s| \approx L$, so one can say that virtual quanta travel at a speed $v \approx 1$.

For solving it at any coupling, it is convenient to switch to the differential equation

$$
\frac{\partial^{2} \Gamma}{\partial S \partial T}=\frac{\lambda / 4 \pi^{2}}{(S-T)^{2}+L^{2}} \Gamma(S, T) .
$$

and change variables to $x=(S-T) / L$ and $y=(S+T) / L$ through

$$
\Gamma(x, y)=\sum_{m} \mathbf{C}_{m} \gamma_{m}(x) e^{\omega_{m} y / 2}
$$

with the corresponding boundary condition $\Gamma(x,|x|)=1$. The dependence of the kernel $\Gamma$ on the relative times $x$ follows from the differential equation

$$
\left(-\frac{d^{2}}{d x^{2}}-\frac{\lambda / 4 \pi^{2}}{x^{2}+1}\right) \gamma_{m}(x)=-\frac{\omega_{m}^{2}}{4} \gamma^{m}(x)
$$


For large $\lambda$ the dominant part of the potential in (32) is from small relative times $x$ resulting into a harmonic equation [27]

$$
\begin{aligned}
& \left(-\frac{d^{2}}{d x^{2}}+\frac{1}{2}\left(\lambda / 4 \pi^{2}\right) x^{2}\right) \gamma_{m}(x) \\
& =-\frac{1}{4}\left(\omega_{m}^{2}-\lambda / \pi^{2}\right) \gamma_{m}(x) .
\end{aligned}
$$

This shows that the sum of the ladders grow exponentially. At large times $T$, the kernel is dominated by the lowest harmonic mode of (33). For large times $S \approx T$ that is small $x$ and large $y$

$$
\Gamma(x, y) \approx \mathbf{C}_{0} e^{-\sqrt{\lambda} x^{2} / 4 \pi} e^{\sqrt{\lambda} y / 2 \pi}
$$

From (4.2) it follows that in the strong coupling limit the ladder generated potential is

$$
V_{\text {lad }}(L)=-\frac{\sqrt{\lambda} / \pi}{L}
$$

which has the same parametric form as the one derived from the AdS/CFT correspondence except for the overall coefficient ${ }^{15}$.

The results of ref. [27] discussed above indicate that summing ladders get some vacuum physics but not all since the overall coefficient is not reproduced exactly. The same conclusion follows from the fact that the expectation values of Wilson lines are gauge invariant, while the ladder diagrams are not. Therefore, some non-ladder diagrams must be equally important and should be included.

Let me skip discussion of any higher order diagrams, summarizing what we have learned about the ladder re-summation which are worth stressing. In these diagrams the transverse jumps of the interaction points would be as small as the time steps namely,

$$
\delta \mathbf{x}_{t} \sim L / \lambda^{1 / 4}
$$

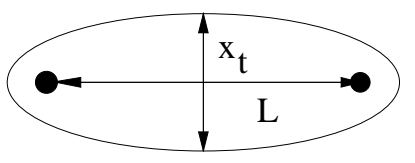

Figure 12: Distribution of the interaction vertices in space. The black circles are static charges. direction must be proportional to $L$ since the conformal symmetry prohibits any other dimensional scale to be developed. Still, in strong coupling the ellipsoid is very elongated $\mathbf{x}_{t} \approx L / \lambda^{1 / 4} \ll L$ : this is what we meant by a "quasi-string" regime in the title of this subsection.

\footnotetext{
${ }^{15}$ Note that the difference is not so large, since $1 / \pi=0.318$ is larger than the exact value 0.228 but about $30 \%$. So additional screening by about $1 / 3$ is needed to get it right. This is of course in the left out higher order diagrams.
} 


\subsection{Bound states of the quasiparticles}

The simplest bound state problem in our case is that of a scalar in the presence of an infinitely heavy source with compensating color charge. The latter acts as an overall attractive Coulomb potential $V$ (the effects of screening will be discussed below). In strong coupling $V$ acts on the accompanying relativistic gluino quasi-instantaneously. For a spherically symmetric potential, the Klein-Gordon equation reads

$$
-\frac{d^{2}}{d r^{2}} \chi_{l}=\left[(E-V)^{2}-m^{2}-\frac{\tilde{l}^{2}}{r^{2}}\right] \chi_{l}
$$

with the wave function $\phi=Y_{l m} \chi_{l}(r) / r$ and the orbital quantum number $\tilde{l}^{2}=l(l+1)^{16}$. For a Coulomblike potential, the equation is exactly solvable in terms of hyper-geometric functions, from which the quantized energy levels are

$$
E_{n l}= \pm m\left[1+\left(\frac{C}{n+1 / 2+\sqrt{\tilde{l}^{2}-C^{2}}}\right)^{2}\right]^{-1 / 2}
$$

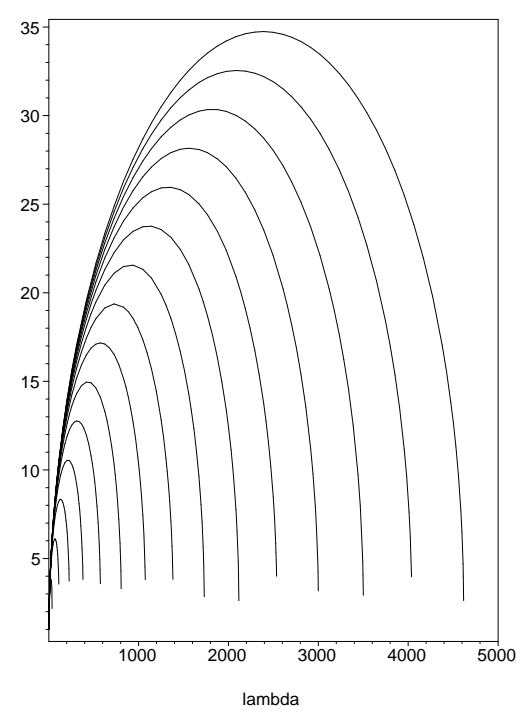

Figure 13: The spectrum of the states versus the 'tHooft coupling constant $\lambda$. for the levels with fixed $n_{r}=0$ and the orbital momentum $l=1 . .15$. One can see that there are light bound states at any coupling.

In weak coupling $C=g^{2} N=\lambda$ is small and the bound states energies are close to $\pm m$. Specifically ${ }^{17}$

$$
E_{n l}=m-\frac{C^{2} m}{2(n+l+1)^{2}},
$$

which is the known Balmer formula. All of that, including the expression above, was known since 1930's.

New view on this formula, in the (opposite) strong coupling limit, gives the following. If the Coulomb law coefficient is large $C=\left(4 \pi^{2} / \Gamma(1 / 4)^{4}\right) \sqrt{\lambda} \gg 1$, the quantized energies are imaginary unless the square root gets balanced by a sufficiently large angular momentum. In this regime, one may ignore the 1 in (37) and obtain the equi -distant spectrum of deeply bound states

$$
E_{n l} \approx \frac{m}{C}\left[(n+1 / 2)+\left((l+1 / 2)^{2}-C^{2}\right)^{1 / 2}\right]
$$

Since the mass is related to the thermal loop with $m \approx \sqrt{\lambda}$, the ratio $m / C \approx T \lambda^{0}$ is proportional to temperature but independent of the coupling constant. More details on the energy dependence on the coupling constant are shown in Fig. 13 for different values of the orbital quantum number $l$ (a) and radial quantum number $n$ (b). All lines end when the Coulomb attraction is able to overcome the centrifugal repulsion.

After we established the spectrum, let us see its consequences for thermodynamics and kinetics at strong coupling. Normally all kinetic quantities are related with scattering cross sections, and the absence of any coupling is a priori implausible physically. In general, thermodynamical quantities count degrees of freedoms and one may think that the coupling constant

\footnotetext{
${ }^{16}$ For a semiclassical analysis at small $l$ one can use the Langer prescription $l(l+1) \rightarrow(l+1 / 2)^{2}$, which is known to yield semiclassically stable S-states. However we will only need large $l$.

${ }^{17}$ The fact that only the combination $n+l$ appears, i.e. principle quantum number, is a consequence of the known Coulomb degeneracy. This is no longer the case in the relativistic case.
} 
may indeed be absent. However, the effective masses of the quasiparticles and all pairwise interactions are still proportional to $\sqrt{\lambda}$ and in general $\lambda^{\alpha}$ with $\alpha>0$. If so, the quasiparticle contributions to the statistical sum should be exponentially small at strong coupling, of the order of $e^{-\lambda^{\alpha}} \ll 1$, and one will have to conclude that some other degrees of freedom are at play.

Indeed there are new degrees of freedom at play in the strong coupling regime of the thermal Coulomb phase. As we have shown above, for large $\lambda$ there are light (deeply bound) binary composites, with masses of order $T$ irrespective of how strong is $\lambda$. The composites are almost point like with thermal sizes of the order of $1 / T$, which readily explains the liquid-like kinetic behavior. The thermal Coulomb phase is a liquid of such composites. The composites are light and should dominate the long-distance behavior of all the finite temperature Euclidean correlators.

We start by explaining the leading contribution to free energy from the gauge theory point as a liquid of composites. The factor of $N_{c}^{2}$ in front of the free energy and the viscosity for instance, follows from the fact that all our composites are in the adjoint representation. Indeed, for fundamental charges the composites are meson-like (color neutral) with all color factors absorbed in the coupling constant. For adjoint charges, there is in addition two spectator charges ${ }^{18}$ that do not participate into the binding. The Coulomb phase is not confining. Thus the number of light and Coulomb bound composites is $N_{c}^{2}$.

The astute reader may raise the question that by now our arguments are totally circular: we have started with $N_{c}^{2}$ massless relativistic states and we have returned to $N_{c}^{2}$ light composites. So what is the big deal? Well, the big deal is that we are in strong coupling, and in fact the degrees of freedom completely reorganized themselves in composites, for otherwise they become infinitely heavy thermally and decouple. This is how Coulomb's law negotiates its deeds in a system with very large charges.

The composites carry large angular momentum in strong coupling, i.e. $l \approx \sqrt{\lambda}$. Their weight contribution to the partition function is of the order of

$$
\int_{l_{\min }}^{l_{\max }} d l^{2}=l_{\max }^{2}-l_{\min }^{2} \approx \lambda^{0}
$$

which is independent of $\lambda$, since the WKB orbits are stable only for $l \approx(\sqrt{\lambda}+1 / \sqrt{\lambda})$. The ensuing thermodynamical sum over the radial quantum number $n$ is independent of $\lambda$, leading to a free energy $\mathbf{F}=\mathbf{C} N_{c}^{2} T$ in agreement with strong coupling result. For weak coupling $\mathbf{C}=1$ while for strong coupling $\mathbf{C}=3 / 4$.

Let us now summarize this section. We have exploited the fact that $N=4 \mathrm{SYM}$ is in a Coulomb phase at all couplings, to argue that in strong coupling (Maldacena regime) color charges only communicate over very short period of times $t \approx L / \lambda^{1 / 4}$ for a fixed separation $L$. This physical observation is enough to show why ladder-like diagrams in the gauge theory reproduce the modified Coulomb's law obtained by the AdS/CFT correspondence using classical gravity.

These observations are generic and suggest that in the gauge theory the modified Coulomb potential applies equally well to relativistic and non-relativistic charges. Indeed, since the relativistic particles move with velocity $v \approx 1$, the color reordering encoded in the modified Coulomb potential goes even faster through a virtual quantum exchange with velocity $v \approx \lambda^{1 / 4} \gg 1$. At strong coupling, the color charge is so large that color rearrangement is so prohibitive unless it is carried instantaneously. This is the only way Coulomb's law could budget its energy. The same observations extend to finite temperature where we have shown that the modified Coulomb potential acquires a screening length of the order of $1 / T$ irrespective of how strong is the coupling.

We have analyzed the effects of a (supercritical) Coulomb field on the motion of colored relativistic particles. Bound states form whenever the squared Coulomb potential balances the effects of centrifugation. Rather unexpectedly, we have found that even though the trajectory of any particular Coulomb

\footnotetext{
${ }^{18}$ For example, a composite can be initially made of blue-red and red-green colors, with red rapidly changing inside the ladder.
} 
bound state depends critically on the coupling $\lambda$, their average density remains about $\lambda$-independent constant. We hope, this explains puzzling results obtained using the string theory.

\section{$5 \quad$ Strongly coupled trapped atoms}

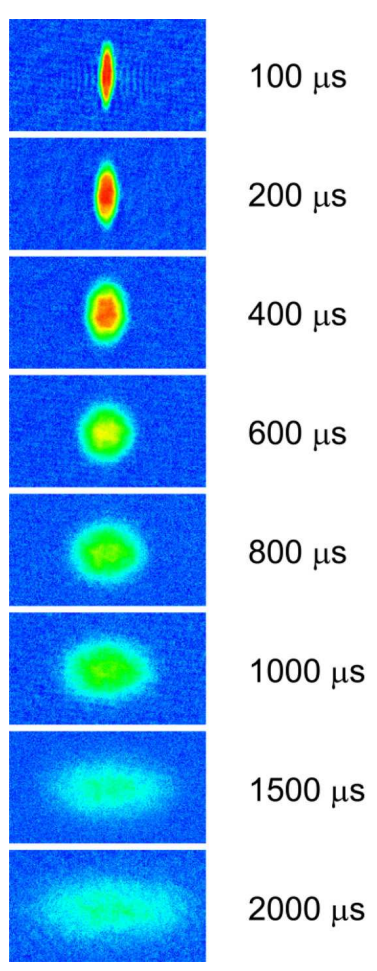

Figure 14: Hydrodynamical elliptic flow of trapped $L i^{6}$ at strong coupling, from [28].

Experimental studies of strongly coupled many-body problems, in which the binary scattering length diverges, are recently done with trapped atomic $L i$ atoms. The scattering length can be tuned till practically infinite values, plus or minus, by applying a magnetic field which shifts the Feshbach resonances.

Remarkably, for its fermionic version it was indeed found very recently that a strong coupling leads to a hydrodynamical behavior [28]. The way it was demonstrated is precisely the same "elliptic flow" as discussed above. One can start with a deformed trap. Normally the gas is so dilute $a n^{1 / 3} \ll 1$ that atoms just fly away isotropically, but when tuning to strong coupling regime is done the expansion is anisotropic and can be described hydrodynamically.

A number of other spectacular experimental discoveries with trapped $L i^{6}$ were also made later. It was found [29] that an adiabatic crossing through the resonance converts nearly all atoms into very loosely bound (but remarkably stable) "Cooper pairs", which can also Bose-condense [30] if the temperature is low enough.

Since in heavy ion collisions the system also crosses the zero binding lines adiabatically, various bound pairs of quarks and gluons should also be generated this way. One might think that such states are pre-prepared at crossing of the zero binding lines, not at the critical line.

\section{Brief summary}

One of the main lessons we have learned from RHIC data is that the QGP we found is very far from a weakly interacting gas of quasiparticles. Although the EoS fitted by hydrodynamics agrees well with lattice data, its transport properties is very different. So, whatever it takes, we should learn more about more strongly coupled systems.

One consequence of stronger coupling is appearance of bound states of quasiparticles. As emphasized by Zahed and myself 2, near the zero binding lines one may indeed expect large rescattering. The experience with trapped cold atoms support this mechanism of hydro very strongly.

Another super-strongly interacting system is $\mathcal{N}=4$ SUSY YM theory: it also leads to hydrodynamical behavior and small viscosity. And again, the bound states seem to be present [4] and may play a key role.

\section{References}

[1] E V Shuryak, Zh.E.T.F 74 (1978) 408, (Sov. Phys. JETP 47 (1978) 212), Phys.Lett. B78 (1978) 150, Yadernaya Fizika 28 (1978) 796, Phys.Rep. 61 (1980) 71.

[2] E. V. Shuryak and I. Zahed, arXiv:hep-ph/0307267, Submitted to PRL

[3] G.E. Brown, C-H. Lee, M. Rho, E.V. Shuryak, hep-ph/0312175 
[4] E. Shuryak and I. Zahed, arXiv:hep-th/0308073. PRD, in press.

[5] E. V. Shuryak and 0. V. Zhirov, Phys.Lett. B89 (1979) 253

[6] L. D. Landau, Izv. Akad Nauk SSSR, ser. fiz. 17 (1953) 51. Reprinted in Collected works by L.D.Landau.

[7] J. Bjorken, Phys. Rev. D27(1983)140

[8] P. J. Siemens and J. 0. Rasmussen, Phys. Rev Lett. 42(1979)880

[9] C. M. Hung and E. V. Shuryak, Phys. Rev. Lett. 75, 4003 (1995) hep-ph/9412360. Phys. Rev. C57, 1891 (1998).

[10] D. Teaney, J. Lauret and E.V. Shuryak, Phys. Rev. Lett. 86 (2001) 4783. P.F. Kolb, P.Huovinen, U. Heinz, H. Heiselberg, Phys. Lett. B500 (2001) 232.

[11] P. F. Kolb and R. Rapp, Phys. Rev. C 67, 044903 (2003) arXiv:hep-ph/0210222.

[12] D. Molnar and M. Gyulassy, Nucl. Phys. A697 (2002) 495; [Erratum-ibid. A703 (2002) 893]

[13] D.Teaney nucl-th/0301099

[14] P.Arnold, G.D.Moore, L.G.Yaffe JHEP 0011 (2000) 001, JHEP 0305, 051 (2003) hep-ph/0302165.

[15] G. Policastro, D. T. Son and A. O. Starinets, Phys. Rev. Lett. 87 (2001) 081601.

[16] P. Kovtun, D. T. Son and A. O. Starinets, JHEP 0310, 064 (2003), hep-th/0309213. A.Buchel and J.Liu, hep-th/0311175.

[17] T. Matsui and H. Satz, Phys. Lett. B178 (1986) 416.

[18] F. Karsch, M. T. Mehr and H. Satz, Z. Phys. C37 (1988) 617.

[19] S. Datta, F. Karsch, P. Petreczky and I. Wetzorke, hep-lat/0208012. M. Asakawa and T. Hatsuda, arXiv:hep-lat/0309001.

[20] Urs M. Heller, F. Karsch, J. Rank, Phys. Rev. D57 (1998) 1438.

[21] T. Schafer and E. V. Shuryak, Phys. Lett. B356 (1995) 147.

[22] P. Petreczky, F. Karsch, E. Laermann, S. Stickan, I. Wetzorke, Nucl. Phys. Proc. Suppl. 106 (2002) 513.

[23] H. A. Weldon, Phys. Rev. D61 (2000) 036003.

[24] G.T. Horowitz and A. Strominger, Nucl. Phys. B360 (1991) 197. S.S.Gubser, I.R.Klebanov and A.A. Tseytlin, Nucl. Phys. B534 (1998) 202

[25] J.M. Maldacena, Adv.Theor.Math.Phys.2:231-252,1998,hep-th/9711200.

[26] S.-J. Rey, S.Theisen and J.-T. Yee,Nucl.Phys.B527:171-186,1998, hep-th/9803135

[27] G. W. Semenoff and K. Zarembo, Nucl. Phys. Proc. Suppl. 108, 106 (2002), hep-th/0202156

[28] K.M.O'Hara et al, Science 298,2179, 2002; T.Bourdel et al, Phys.Rev.Lett.91 (2003) 020402.

[29] J.Cubizolles et al, cond-mat/0308018, K.Strecker et al cond-mat/0308318

[30] M.W. Zwierlein et al, cond-mat/0311617 